\title{
Curvature Energy and Their Spectrum in the Spinor-Twistor Framework: Torsion as Indicium of Gravitational Waves
}

\author{
Francisco Bulnes' ${ }^{1}$, Yuri Stropovsvky², Igor Rabinovich ${ }^{2}$ \\ ${ }^{1}$ Research Department in Mathematics and Engineering, TESCHA, IINAMEI, Chalco, Mexico \\ ${ }^{2}$ Mathematics Department, Lomonosov Moscow State University, Moscow, Russia \\ Email: francisco.bulnes@tesch.edu.mx
}

How to cite this paper: Bulnes, F., Stropovsvky, Y. and Rabinovich, I. (2017) Curvature Energy and Their Spectrum in the Spinor-Twistor Framework: Torsion as Indicium of Gravitational Waves. Journal of Modern Physics, 8, 1723-1736. https://doi.org/10.4236/jmp.2017.810101

Received: August 3, 2017

Accepted: September 9, 2017

Published: September 12, 2017

Copyright $\odot 2017$ by authors and Scientific Research Publishing Inc. This work is licensed under the Creative Commons Attribution International License (CC BY 4.0). http://creativecommons.org/licenses/by/4.0/

\begin{abstract}
The twistor kinematic-energy model of the space-time and the kinematic-energy tensor as the energy-matter tensor in relativity are considered to demonstrate the possible behavior of gravity as gravitational waves that derive of mass-energy source in the space-time and whose contorted image is the spectrum of the torsion field acting in the space-time. The energy of this field is the energy of their second curvature. Likewise, it is assumed that the curvature energy as spectral curvature in the twistor kinematic frame is the curvature in twistor-spinor framework, which is the mean result of this work. This demonstrates the lawfulness of the torsion as the indicium of the gravitational waves in the space-time. A censorship to detect gravitational waves in the space-time is designed using the curvature energy.
\end{abstract}

\section{Keywords}

Censorship Condition, Contorted Surface, Curvature Energy, Gravitational Waves, Matter-Energy Tensor, 3-Dimensional Sphere, Spinor Fields, Twistor Kinematic-Energy Model, Weyl Curvature

\section{Introduction}

The twistor kinematic energy model could establish to the future-null-infinity in the space-time, a quasi-local matter model represented through gravitational waves of cylindrical type considering the condition on the spinor fields respective, in the null-infinity. Here, is obtained the asymptotical flat space-time far away of the mass-energy source.

We consider the Penrose's definition of the kinematic twistor associated to a 
2-surface in a general curved space when the total momentum of energy and angular momentum to a system in special relativity and in linearized general relativity can be characterized geometrically. Of fact, the geometrical evidence of torsion through a contorted surface is wanted.

We consider a source as total charge depending of $k^{\mathrm{a}}$, (Killing vector) of the Minkowski space background, modeled this as $M \cong S^{2} \otimes C^{2} \otimes M$, which has an important analytic system of twistor solution in the same space-time $\mathrm{M} \cong \mathrm{S}^{+} \oplus \mathrm{S}^{-}$. Then their system has a complex 4-dimensional solutions family $\left(\cong C^{2}\right)$ and the family defines the 2-surface twistor space $\pi(S)$.

But, in a surface of arbitrary genus $g$, and of index $4(1-g)$, the solution is a general twistor solution, which can be given though a model problem between bosonic fields deduced of the dual problem given by the relation:

$$
\mathrm{A}_{\alpha \beta} \mathrm{I}^{\beta \gamma} \Sigma_{\gamma \alpha^{\prime}}=\mathrm{A}_{\alpha^{\prime} \gamma} \mathrm{I}^{\beta^{\prime} \gamma^{\prime}} \Sigma_{\gamma^{\prime} \alpha},
$$

to the energy-matter tensors $\mathrm{T}^{a b}$, and the integral solution given to the kinematic tensor $\mathrm{A}_{\alpha \beta}$, through the energy-matter tensor,

$$
\mathrm{A}_{\alpha \beta} \mathrm{Z}_{1}^{\alpha} \mathrm{Z}_{2}^{\beta}=\frac{1}{4 \pi \mathrm{G}} \int_{\mathrm{S}} \mathrm{R}_{\mathrm{abcd}} \mathrm{f}^{\mathrm{ab}} \mathrm{d} \sigma^{\mathrm{cd}}=\int_{\Sigma} \mathrm{T}_{\mathrm{ab}} \mathrm{k}^{\mathrm{a}} \mathrm{d} \sigma^{\mathrm{b}},
$$

The exhibition of the kinematic tensor happens when the special surface inside space-time background $M$, results to be the product $S^{+} \otimes S^{-}$, of the twistor 2-surface $\pi\left(S^{1}\right)$, and also (2) defines a kinematic twistor tensor $\mathrm{A}_{\alpha \beta}$, as element of this symmetrized product of two 2-surfaces

$\mathrm{A}_{\alpha \beta} \in(\pi(S) \otimes \pi(S))^{*}$, which is a twistor space of (valence-2) and symmetric dual twistor.

Proposition 1.1. The twistor kinematic tensor $\mathrm{A}_{\alpha \beta}$, is an element in duality of the energy-mass tensor $\mathrm{T}^{\mathrm{ab}}$.

We observe the following figure establishing the duality signed in the proposition 1.1. (see Figure 1).

Proof. Their demonstration is given considering the relation $\mathrm{A}_{\alpha \beta} \mathrm{I}^{\beta \gamma} \Sigma_{\gamma \alpha^{\prime}}=\mathrm{A}_{\alpha^{\prime} \gamma} \mathrm{I}^{\beta \gamma^{\prime}} \Sigma_{\gamma^{\prime} \alpha}$, where the second member can be had as a spinor using the integral (2):

$$
\mathrm{A}_{\alpha \beta} \mathrm{Z}_{1}^{\alpha} \mathrm{Z}_{2}^{\beta}=\frac{1}{4 \pi \mathrm{G}} \int \mathrm{R}_{\mathrm{ABcd}} \omega_{1}^{\mathrm{A}} \omega_{2}^{B} \mathrm{~d} \sigma^{\mathrm{cd}},
$$
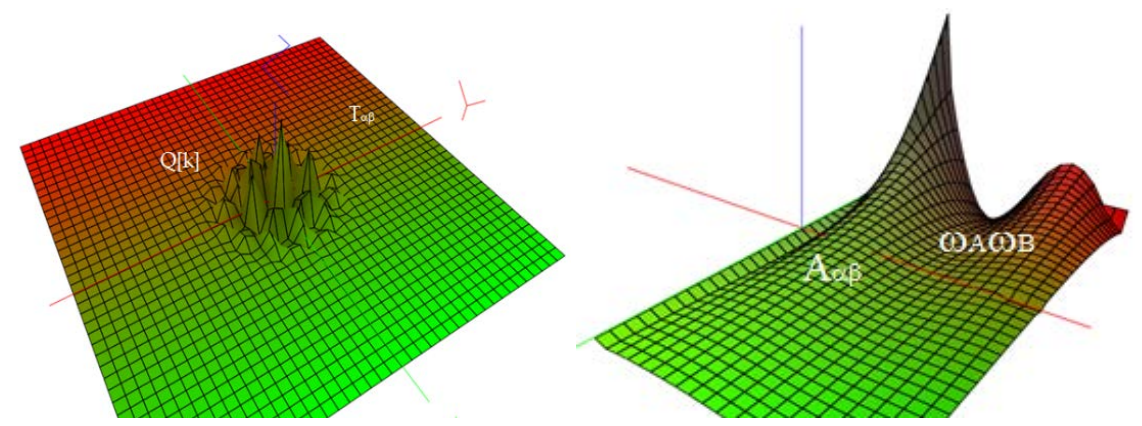

Figure 1. Duality between tensors $A_{\alpha \beta}$, and $T^{a b}$. 
which, using the spinor framework [1] [2] inside the integral (3) we have:

$$
\mathrm{A}_{\alpha \beta} \mathrm{Z}_{1}^{\alpha} \mathrm{Z}_{2}^{\beta}=\frac{1}{4 \pi \mathrm{G}} \int\left\{\left(\phi_{01}-\psi_{1}\right) \omega_{1}^{\mathrm{A}} \omega_{2}^{\mathrm{B}}+\left(\phi_{11}+\Lambda-\psi_{2}\right)\left(\omega_{1}^{0} \omega_{2}^{1}+\omega_{1}^{1} \omega_{2}^{0}\right)+\left(\phi_{21}-\psi_{3}\right) \omega_{1}^{1} \omega_{2}^{1}\right\} \mathrm{d} \mathrm{S},
$$

which is simplified using the spinor frame equations ${ }^{1}$ :

$$
\begin{aligned}
& \wp \pi_{0^{\prime}}+\rho \pi_{1^{\prime}}=\mathrm{i}\left(\psi_{2}-\phi_{11}-\Lambda\right) \omega^{1}+\mathrm{i}\left(\psi_{1}-\phi_{01}\right) \omega^{0}, \\
& \wp^{\prime} \pi_{1^{\prime}}+\rho^{\prime} \pi_{0^{\prime}}=\mathrm{i}\left(\psi_{3}-\phi_{21}\right) \omega^{1}+\mathrm{i}\left(\psi_{2}-\phi_{11}-\Lambda\right) \omega^{0},
\end{aligned}
$$

to the integral

$$
\mathrm{A}_{\alpha \beta} \mathrm{Z}_{1}^{\alpha} \mathrm{Z}_{2}^{\beta}=\frac{-\mathrm{i}}{4 \pi \mathrm{G}} \int\left(\pi_{0}^{1} \pi_{1^{\prime}}^{2}+\pi_{1^{\prime}}^{1} \pi_{0^{\prime}}^{2}\right) \mathrm{dS},
$$

which establishes the required duality.

Of the integral involved in (6), we note that the twistor kinematic tensor $\mathrm{A}_{\alpha \beta}$, depends of $\mathrm{S}$, which has more mean, that is to say, depends on the first and second fundamental forms of $S$.

This means the presence of curvature inside spinor terms in the integrating of (6). This explains only the dependence of the energy due to curvature. Then to spinor fields of the form $\left(\omega^{\mathrm{A}}, \pi_{\mathrm{A}^{\prime}}\right)$, we have the quantity [1]:

$$
\Sigma=\omega^{\mathrm{A}} \bar{\pi}_{\mathrm{A}^{\prime}}+\bar{\omega}^{\mathrm{A}^{\prime}} \pi_{\mathrm{A}^{\prime}},
$$

which is constant to constant curvature space. However, for a 2-surface in a general space-time $\mathbb{M}$, there is no reason to that (7) could be constant. Likewise, we have the following proposition:

Proposition 1.2. (7) is constant for every 2-surface twistor if and only if the 2-surface with their field $\left(\omega^{\mathrm{A}}, \pi_{\mathrm{A}^{\prime}}\right)$, is embedded in a conformally flat spacetime modulo certain genericity conditions.

Then in little words, the proposition 1.2. prepares a detection condition from a contorted property of the 2-surface when is affected by the presence of a field source. This in the conformally conditions detects curvature which is measured and modeled in the spinor waves as is showed in Figure 1 in the 2-surface twistor of the twistor kinematic tensor $\mathrm{A}_{\alpha \beta}$. In our study of spectral curvature we can define this measure as curvature energy obtained through twistor frame of the energy-mass tensor, as in the integrals (3) and (6). These have involved a curvature tensor, which has curvature energy as spinor field energy or spinor wave, this last understood as energy manifestation in the kinematic tensor space $(\pi(\mathrm{S}) \otimes \pi(\mathrm{S}))^{*}$.

Likewise, the curvature energy as spectral curvature in the twistor kinematic frame is the curvature in twistor-spinor framework.

\footnotetext{
${ }^{1}$ The twistor equations to valence-2 on symmetric spinor $\omega^{\mathrm{AB}}$, can be written as:
}

$$
\nabla_{\mathrm{A}^{\prime}}^{\mathrm{A}} \omega^{\mathrm{BC}}=-\mathrm{i} \in^{\mathrm{A}(\mathrm{B}} \mathrm{k}_{\mathrm{A}^{\prime}}^{\mathrm{C})},
$$

which has a 10-dimensional complex solutions space. Their solution space is spanned by fields $\omega^{\mathrm{AB}}$, of the form $\omega_{1}^{(\mathrm{A}} \omega_{2}^{\mathrm{B})}$, (such and is showed in Figure 1), where each $\omega_{\mathrm{i}}^{\mathrm{A}}$, satisfies the twistor equation

$$
\nabla_{\mathrm{A}}^{\mathrm{A}} \omega^{\mathrm{B}}=-\mathrm{i} \in^{\mathrm{AB}} \pi_{\mathrm{A}} \text {, }
$$

whose solutions defines a 4-dimensional complex vector space which is the twistor space $\pi$. 
Def. 1.1. A 2-surface $S$, is contorted if their embedding involves one component of the twistor space $\pi(S)$.

Remember that $\mathrm{A}_{\alpha \beta}$, is the twistor kinematic tensor of the given source. Due to that the twistor equations to spinor fields $\omega^{\mathrm{AB}}$, have a 10-dimensional complex solution space, $\mathrm{A}_{\alpha \beta}$, apparently has too much information in it. To curvature we want solutions provided of the energy-mass tensor. Then for simplification and the spinor framework is obtained the linearized general relativity context where the tensor $\mathrm{T}^{\mathrm{ab}}$, must satisfy the equation

$$
\nabla^{\mathrm{a}} \mathrm{T}^{\mathrm{ab}}=0,
$$

thought out as a source for a linearized gravitational field. This will bring a linearized Riemann tensor, which will be agreed to the spinor frame considering the components $f_{a b}=\omega_{A B} \in_{A^{\prime} B^{\prime}}$, which relates the spinor field $\omega_{\mathrm{AB}}$, with the Killing vector $k^{\mathrm{a}}$, in the twistor equations to twistors of valence-2. Then using divergence theorem when $S$, is a 2 -surface on the 3 -surface $\Sigma$, as given in (7) surrounding the source, we have several censorship conditions designed through dominating energy condition of curvature.

Then the energy of the twistor kinematic tensor that will the energy substantive to measure curvature energy in the case of the twistor-spinor framework, is given in energy domain $\mathrm{M}_{\mathrm{N}} \geq \mathrm{A}_{\alpha \beta} \mathrm{Z}^{\alpha} \mathrm{I}^{\beta \gamma} \overline{\mathrm{Z}}_{\gamma} \geq 0$.

The inequality written in the last paragraph conforms the inequalities family of Hilbert type in twistor theory required to explain the range or domain of energy where can be censed the existence of the massive object that will produce the torsion of the space. Then of this torsion will be produced the gravitational waves in the space-time far of the massive source, but whose asymptotic behavior helps to the understanding the post-Newtonian limit after of the horizon of events of the massive source, when the space-time tends to de Sitter Universe.

\section{Curvature and Twistor-Spinor Framework}

A result of the curvature digression as an observable of an object obtained through integral transform on cycles is the following theorem.

Theorem 2. 1 (Y. Stropovsvky, F. Bulnes, I. Rabinovich). We consider the embedding $\sigma: \Sigma \rightarrow(\ulcorner(S) \otimes\urcorner(S))^{*}$. The space $\sigma(\Sigma)$, is smoothly embedded in the twistor space $(\pi(S) \otimes \pi(S))^{*}$. Then their curvature energy is the energy given in the interval $\mathrm{M}_{\mathrm{N}} \geq \mathrm{A}_{\alpha \beta} \mathrm{Z}^{\alpha} \mathrm{I}^{\beta \gamma} \bar{Z}_{\gamma} \geq 0$.

Some considerations on the curvature of twistor-spinor framework in a complex Riemannian manifold are necessary to clarify. After we realize the demonstration of the theorem 2. 1, which is the central goal of the chapter.

We consider the twistor fields $\Psi_{\mathrm{ABCD}}$, and $\tilde{\Psi}_{\mathrm{A}^{\prime} \mathrm{B}^{\prime} \mathrm{C}^{\prime} \mathrm{D}^{\prime}}$, satisfying the twistor equations

$$
\nabla^{\mathrm{AA}} \Psi_{\mathrm{ABCD}}=0, \nabla^{\mathrm{AA}^{\prime}} \Psi_{\mathrm{A}^{\prime} \mathrm{B}^{\prime} \mathrm{C}^{\prime} \mathrm{D}^{\prime}}=0,
$$

whose solutions are given by the twistor contour integrals 


$$
\Psi_{\mathrm{ABCD}}=\oint_{\partial_{\omega^{2}} 2 \pi=\mathrm{S}^{1}} \frac{\partial}{\partial \omega^{\mathrm{A}}} \frac{\partial}{\partial \omega^{\mathrm{B}}} \frac{\partial}{\partial \omega^{\mathrm{C}}} \frac{\partial}{\partial \omega^{\mathrm{D}}} \mathrm{f}(\mathrm{z}) \mathrm{dz},
$$

and

$$
\tilde{\Psi}_{\mathrm{A}^{\prime} \mathrm{B}^{\prime} \mathrm{C}^{\prime} \mathrm{D}^{\prime}}=\oint_{\partial_{\omega} 2 \pi=\mathrm{S}^{1}} \pi_{\mathrm{A}^{\prime}} \pi_{\mathrm{B}^{\prime}} \pi_{\mathrm{C}^{\prime}} \pi_{\mathrm{D}^{\prime}} \tilde{\mathrm{f}}(\mathrm{z}) \mathrm{dz}
$$

which could be deformed by the presence of an incurved section of the space having the energy-stress-mass tensor condition given by Einstein in conformally. Here $f(z)$, is a function of homogeneous degree +2 , and $\tilde{f}(z)$, is a function of homogeneous degree- $6^{2}$.

We can consider the linearized gravity framework (which can be complex) where we have the curvature, then considering the deforming contributions of the contour integrals given on (10) and (11), we have:

$$
\mathrm{K}_{\mathrm{abcd}}=\Psi_{\mathrm{ABCD}} \in_{\mathrm{A}^{\prime} \mathrm{B}^{\prime}} \in_{\mathrm{C}^{\prime} \mathrm{D}^{\prime}}+\epsilon_{\mathrm{AB}} \in \in_{\mathrm{CD}} \tilde{\Psi}_{\mathrm{A}^{\prime} \mathrm{B}^{\prime} \mathrm{C}^{\prime} \mathrm{D}^{\prime}},
$$

where $\Psi_{\mathrm{ABCD}} \in_{\mathrm{A}^{\prime} \mathrm{B}^{\prime}} \in \in_{\mathrm{C}^{\prime} \mathrm{D}^{\prime}}$, is the anti-self-dual component and $\in_{\mathrm{AB}} \in_{\mathrm{CD}} \Psi_{\mathrm{A}^{\prime} \mathrm{B}^{\prime} \mathrm{C}^{\prime} \mathrm{D}^{\prime}}$, is the self-dual part. Here $\Psi_{\mathrm{ABCD}}$, and $\tilde{\Psi}_{\mathrm{A}^{\prime} B^{\prime} C^{\prime} \mathrm{D}^{\prime}}$, both symmetric if $\mathrm{K}_{\mathrm{abcd}}$, is real, due that $\Psi_{\mathrm{ABCD}}$, and $\tilde{\Psi}_{\mathrm{A}^{\prime} \mathrm{B}^{\prime} \mathrm{C}^{\prime} \mathrm{D}^{\prime}}$, are both complex conjugate.

The differential of the integrals (10) and (11) comes given as:

$$
\delta \mathrm{z}=\epsilon^{\mathrm{A}^{\prime} \mathrm{B}^{\prime}} \pi_{\mathrm{A}^{\prime}} \mathrm{d} \pi_{\mathrm{B}^{\prime}}
$$

Likewise, $\mathrm{f}$, and $\tilde{\mathrm{f}}$, are representatives of cohomology. Here, we have the spectral curvature considering their spectra in the twistor space

$(\pi(S) \otimes \pi(S))^{*}$. But is necessary consider all cases that are presented in the complex Riemannian manifold M, to curvature study. Likewise in general relativity, to the flat space we can consider the duality between the spaces $\mathrm{CM}$, and $\mathrm{CPP}^{3}$, having a null separation dual to meeting lines (see Figure 2).

Also in deformation theory, the anti-self-dual complex space-time has correspondence in duality with the general Ricci-flat space $\mathbb{C P P}^{1}$, where circles of the deformed tube have images in a $\pi$-space. These deformed tubes could be geometrical representations of 2-dimensional superstrings whose circles of their diameter are points of the infinite line or $\pi$-space. Then the anti-self-dual complex space-time and the Ricci-flat space are equivalent to the parallelism for $\pi_{\mathrm{A}}$ - spinors (locally), that is to say,

$$
\left[\nabla_{\mathrm{AA}^{\prime}}, \nabla_{\mathrm{BB}^{\prime}}\right] \pi_{\mathrm{C}^{\prime}}=0,
$$

taking place a curvature classification due the products of the summation indices [1]. Likewise, the curvature in $\Psi_{\mathrm{ABCD}}$, represents the non-existence of holomorphic planes ${ }^{3}$ in the twistor space to the tube (twistor tube) $\mathbb{C} \mathcal{T}$, then is required the twistor component due to the homogeneous degree-6, $\tilde{\Psi}_{\mathrm{A}^{\prime} \mathrm{B}^{\prime} \mathrm{C}^{\prime} \mathrm{D}^{\prime}}$,

${ }^{2}$ Left-handed graviton with $\mathrm{f}$, homogeneous with degree +2 . And right-handed graviton with $\tilde{f}$, homogeneous with degree -6 .

${ }^{3}$ Def. A $\quad \beta$-plane is a holomorphic plane in the twistor space ${ }_{\mathrm{c}} \mathcal{T}$. 


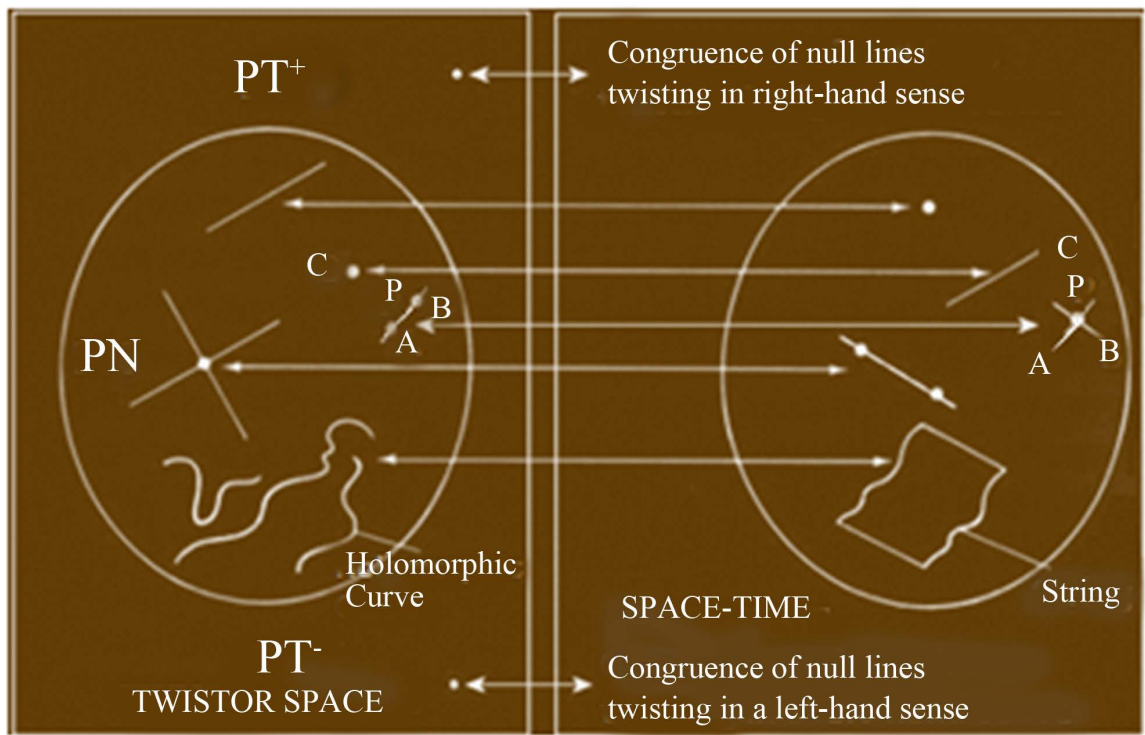

Figure 2. Dualities between the twistor space elements and space-time elements [1].

which involves a torsion energy (second curvature energy) and the Ricci-flat space condition.

The appearing necessity of torsion as special factor of curvature detection in the deforming of the microscopic space-times in $\mathbb{M}$, is a condition of existence of curvature in these spaces. Likewise, in [2] is obtained a particular solution, which could establish curvature in spinor-twistor terms through of the second component of curvature given in (12).

Here the problem is to see the cause of second curvature to $K_{a b c d}$, which are the elements mentioned before.

Let M, a complex Riemannian manifold. We have the following natural conjecture.

Conjecture 2.1. The curvature in spinor-twistor framework can be perceived with the appearing of the torsion and the anti-self-dual fields.

Proof. We consider the complex Minkowski space M. Then their Weil curvature is anti-self-dual given place to the $\alpha$-planes where could to exist distortion or twists. But this exists to a space-time referred to the group $U_{c}(4)$, in gravity. Under these conditions the complex Minkowski space can present a torsion as the candidate to produce distortions as second curvature in the space M, (locally). But the spinor model of torsion can be writte as:

$$
\mathrm{S}_{\mathrm{ab}}{ }^{\mathrm{c}}=\chi_{\mathrm{AA}^{\prime}}^{\mathrm{CC}^{\prime}} \in_{\mathrm{A}^{\prime} \mathrm{B}^{\prime}}+\tilde{\chi}_{\mathrm{A}^{\prime} \mathrm{B}^{\prime}}^{\mathrm{CC} \in_{\mathrm{AB}}},
$$

where spinors $\chi_{\mathrm{A}^{\prime} \mathrm{B}^{\prime}}$, and $\tilde{\chi}_{\mathrm{A}^{\prime} \mathrm{B}^{\prime}}$, are symmetric in $\mathrm{AB}$, and $\mathrm{A}^{\prime} \mathrm{B}^{\prime}$, respectively and linearly independents. Likewise, re-written the spinor equation to torsion (15), in the twistor-spinor framework we have ${ }^{4}$ :

\footnotetext{
${ }^{4} \forall X, Y \in \mathcal{X}$, (M), with connection $\nabla$, we have the torsion expression [3]:

$$
[\mathrm{X}, \mathrm{Y}]=\nabla_{\mathrm{X}} \mathrm{Y}-\nabla_{\mathrm{Y}} \mathrm{X}-2 \mathrm{~S}(\mathrm{X}, \mathrm{Y})
$$
}

If we consider the fields defined as $X^{\mathrm{a}}=\lambda^{\mathrm{A}} \pi^{A^{\prime}}$, and $\mathrm{Y}^{\mathrm{a}}=\eta^{\mathrm{A}} \pi^{A^{\prime}}$, then the twistor-spinor model of torsion is given. 


$$
\pi^{\mathrm{A}^{\prime}}\left(\nabla_{\mathrm{AA}^{\prime}} \pi_{\mathrm{B}^{\prime}}\right)=\xi_{\mathrm{A}} \pi_{\mathrm{B}^{\prime}}-2 \pi^{\mathrm{A}^{\prime}} \pi^{\mathrm{C}^{\prime}} \tilde{\chi}_{\mathrm{A}^{\prime} \mathrm{B}^{\prime} \mathrm{AC}},
$$

Then we must to do that the anti-self-dual complex space-time and the Ricci-flat space are equivalent to the parallelism for $\pi_{\mathrm{A}}$-spinors (locally) that are had with the formalisms (15) and (16). This condition is an integrability condition necessary to the existence of solutions to equation types as (14). Here arise several tensors considering different spinor indices bracket products. Likewise, the curvature tensor written through spinors tools, using the spinor Ricci identities stays as:

$$
\begin{aligned}
& \mathrm{R}_{\mathrm{abcd}}=\phi_{\mathrm{ABCD}} \in_{\mathrm{A}^{\prime} \mathrm{B}^{\prime}} \in \in_{\mathrm{C}^{\prime} \mathrm{D}^{\prime}}+\tilde{\phi}_{\mathrm{A}^{\prime} \mathrm{B}^{\prime} \mathrm{C}^{\prime} \mathrm{D}^{\prime}} \in \in_{\mathrm{AB}} \in_{\mathrm{CD}}+\Phi_{\mathrm{ABC}^{\prime} \mathrm{D}^{\prime}} \in \in_{\mathrm{A}^{\prime} \mathrm{B}^{\prime}} \in \in_{\mathrm{CD}}+\tilde{\Phi}_{\mathrm{A}^{\prime} \mathrm{B}^{\prime} \mathrm{CD}} \in_{\mathrm{AB}} \in \in_{\mathrm{C}^{\prime} \mathrm{D}^{\prime}} \\
& +\Delta_{\mathrm{AB}} \in_{\mathrm{A}^{\prime} \mathrm{B}^{\prime}} \in_{\mathrm{CD}} \in_{\mathrm{C}^{\prime} \mathrm{D}^{\prime}}+\tilde{\Delta}_{\mathrm{A}^{\prime} \mathrm{B}^{\prime}} \in_{\mathrm{AB}} \in_{\mathrm{CD}} \in_{\mathrm{C}^{\prime} \mathrm{D}^{\prime}}+\Omega\left(\epsilon_{\mathrm{AC}} \in_{\mathrm{BD}}+\epsilon_{\mathrm{AD}} \in_{\mathrm{BC}}\right) \in_{\mathrm{A}^{\prime} \mathrm{B}^{\prime}} \in_{\mathrm{C}^{\prime} \mathrm{D}^{\prime}} \\
& +\tilde{\Omega}\left(\in_{\mathrm{A}^{\prime} \mathrm{C}^{\prime}} \in_{\mathrm{B}^{\prime} \mathrm{D}^{\prime}}+\in_{\mathrm{A}^{\prime} \mathrm{D}^{\prime}} \in_{\mathrm{B}^{\prime} \mathrm{C}^{\prime}}\right) \in_{\mathrm{AB}} \in_{\mathrm{CD}} \text {, }
\end{aligned}
$$

and the torsion through integrability condition (15):

$$
\left[\nabla_{\mathrm{C}^{\left(\mathrm{A}^{\prime}\right.}} \nabla_{\left.\mathrm{B}^{\prime}\right)}^{\mathrm{C}}-2 \tilde{\chi}_{\mathrm{A}^{\prime} \mathrm{B}^{\prime}} \nabla_{\mathrm{HH}^{\prime}}\right] \pi^{\mathrm{C}^{\prime}}=\phi_{\mathrm{A}^{\prime} \mathrm{B}^{\prime} \mathrm{E}^{\prime}}^{\mathrm{C}^{\prime}} \pi^{\mathrm{E}^{\prime}}-2 \tilde{\Omega} \pi_{(\mathrm{A}} \in_{\left.\mathrm{B}^{\prime}\right)} \mathrm{C}^{\prime}+\tilde{\Delta}_{\mathrm{A}^{\prime} \mathrm{B}^{\prime}} \pi^{C^{\prime}},
$$

where is clear the appearing of torsion in the terms $\phi_{\mathrm{A}^{\prime} \mathrm{B}^{\prime} \mathrm{E}^{\prime}}^{\mathrm{C}^{\prime}} \pi^{\mathrm{E}^{\prime}}-2 \tilde{\Omega} \pi_{(\mathrm{A}} \in_{\left.\mathrm{B}^{\prime}\right)} \mathrm{C}^{\prime}$, and the integrability condition to $\alpha$-surfaces also is appeared considering $\lambda_{\mathrm{A}} \lambda^{\mathrm{A}}=\pi_{\mathrm{A}^{\prime}} \pi^{\mathrm{A}^{\prime}}=0$.

Then a total spinor field $\Psi$, that detect distortions due to curvature existence in the microscopic level can be written as:

$$
\Psi=\oint_{\mathrm{S}^{1}} \frac{\partial}{\partial \omega^{\mathrm{A}}} \frac{\partial}{\partial \omega^{\mathrm{B}}} \frac{\partial}{\partial \omega^{\mathrm{C}}} \frac{\partial}{\partial \omega^{\mathrm{D}}} \in_{\mathrm{AB}} \in_{\mathrm{BC}} \pi \mathrm{d} \pi+\oint_{\mathrm{S}^{1}} \pi_{\mathrm{A}} \pi_{\mathrm{B}} \pi_{\mathrm{C}} \pi_{\mathrm{D}} \in_{\mathrm{A}^{\prime} \mathrm{B}^{\prime}} \in_{\mathrm{B}^{\prime} \mathrm{C}^{\prime}} \mathrm{d} \pi,
$$

where are perceived these distortions with right-handed gravity (see Figure 3).

Then we have the combining of two deformations with one component with two interaction planes. Likewise, in both components are considered the spinor fields $\Psi_{\mathrm{ABCD}}$, and $\tilde{\Psi}_{\mathrm{ABCD}}$, where the component $\tilde{\Psi}_{\mathrm{ABCD}}$, is really the principal contribution of the distortions:

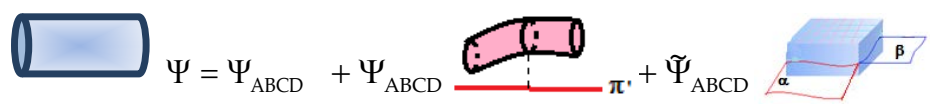

But in the component $\Psi_{\mathrm{ABCD}}$, also happens certain distortion understood as twistor waves with image in spinors, where to the twistor function $f(z)$, the degree +2 has the infinitesimal shunt to wave-spinor $\hat{\omega}^{\mathrm{A}}=\omega^{\mathrm{A}}+\in \eta^{\mathrm{AB}} \frac{\partial \mathrm{f}}{\partial \omega^{\mathrm{B}}}$, and $\hat{\pi}_{\mathrm{A}}=\pi_{\mathrm{A}}$, with vector field $\eta^{\mathrm{AB}} \frac{\partial \mathrm{f}}{\partial \omega^{\mathrm{B}}} \frac{\partial}{\partial \omega^{\mathrm{A}}}$, agreeing with the integral:

$$
\Psi_{\mathrm{ABCD}}=\oint \frac{\partial}{\partial \omega^{\mathrm{A}}} \cdots \frac{\partial}{\partial \omega^{\mathrm{D}}} \pi \mathrm{d} \pi,
$$

Then the field $\tilde{\Psi}_{\mathrm{ABCD}}$, is incorporated as was signed in (20) using this field may be, with the differential form in major dimension. But necessarily has that be incorporated in a 3-dimensional space which is inside an energy state space which will give a censorship condition to the detection and measure of first and 


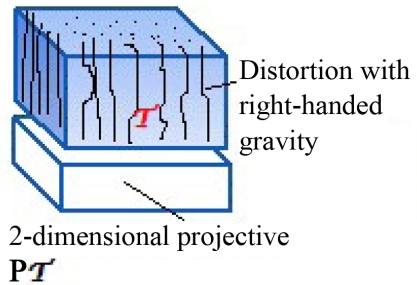

(a)

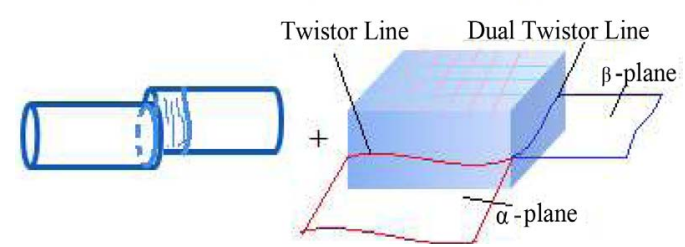

(b)

Figure 3. (a) Distortions with right-handed gravity by spinor $\Psi_{\mathrm{ABCD}} \alpha+\tilde{\Psi}_{\mathrm{ABCD}} \beta$; (b) Distorted tube more right-handed gravity given by (19).

second curvature considering the twistor-spinor waves used in the field framework.

Likewise, with this spirit of ideas, will be necessary incorporate a 3-forms of Sparling type to use the adequate Hamiltonian vector density where their $\mathcal{H}$ -space is equal to ASD space-time whose the non-linear graviton twistor space is the space $\mathbb{P} \mathcal{T}$, of twistor lines $\mathrm{Z}$.

\section{The Kinematic Tensor and the Dominating Energy Condition to Torsion Indicium}

Remember that the wanted positivity condition can be expressed as (using Hermitian matrix):

$$
\mathrm{A}_{\alpha \beta} \overline{\mathrm{I}}_{\bar{\alpha}} \mathrm{Z}^{\alpha} \bar{Z}^{\alpha} \geq 0,
$$

$\forall Z^{\alpha}$, a constant field, that is to say, the Hermitian matrix $\mathrm{A}_{\alpha \beta} \overline{\mathrm{I}}_{\bar{\alpha}}^{\beta}$, could be positive semi-definite.

Likewise, considering that the exhibition of curvature energy can be written through the energy densities obtained for twistor fields and their dual, the spinor frame, we can write the dominating energy condition as the integral:

$$
\begin{aligned}
\mathrm{A}_{\alpha \beta} \overline{\mathrm{I}}_{\bar{\alpha}}^{\beta} \mathrm{Z}^{\alpha} \overline{\mathrm{Z}}^{\alpha} & =\frac{\mathrm{i}}{8 \pi \mathrm{G}} \int_{\mathrm{S}} \bar{\pi}^{\mathrm{A}} \mathrm{d} \pi^{\mathrm{A}^{\prime}} \wedge \theta_{\mathrm{AA}^{\prime}} \\
& =\frac{\mathrm{i}}{8 \pi \mathrm{G}} \int_{\mathcal{H}} \mathrm{d} \bar{\pi}_{\mathrm{A}} \wedge \mathrm{d} \pi_{\mathrm{A}^{\prime}} \wedge \theta^{\mathrm{AA}^{\prime}}-\frac{1}{2} \mathrm{G}_{\mathrm{ab}} \bar{\pi}^{\mathrm{A}} \pi^{\mathrm{A}^{\prime}} \boldsymbol{X}^{\mathrm{b}},
\end{aligned}
$$

$\forall \mathbf{X}^{\mathrm{b}}$, a Hamiltonian vector density:

$$
\mathbf{X}^{\mathrm{b}}=\frac{1}{6} \in_{\mathrm{abc}}^{\mathrm{b}} \theta^{\mathrm{c}} \wedge \theta^{\mathrm{d}} \wedge \theta^{\mathrm{c}},
$$

which is the 3 -form mentioned in Table 1.

In the conformally flat space-time (Ricci-flat space) we have solutions to the equation $\nabla_{\mathrm{A}^{\prime}}^{\mathrm{A}} \omega^{\mathrm{B}}=-\mathrm{i} \in \epsilon^{\mathrm{AB}} \pi_{\mathrm{A}^{\prime}}$, and any 2 -surface twistor arises by restriction of a "4-space-time"such as in the FRW-cohomology". Then the kinematic twistor is again written in terms of the flux integrals.

Considering the scalar "observable" due the twistor kinematic tensor $\mathrm{A}_{\alpha \beta}$, given by, $\mathrm{M}_{\mathrm{N}}$, we have:

${ }^{5}$ Friedman-Robertson-Walker cohomology. 
Table 1. Differential forms to different objects in twistor-spinor theory.

\begin{tabular}{|c|c|c|c|}
\hline \multirow[b]{2}{*}{$\#$} & \multicolumn{3}{|c|}{ Differential Forms } \\
\hline & Physical Object & $\begin{array}{l}\text { N-dimension } \\
\text { al form }\end{array}$ & Locally Expression in M. \\
\hline 1 & Deformed Line & $\begin{array}{l}\text { 1-form: } \\
\ell(=\delta \mathrm{z})\end{array}$ & $\delta \mathrm{Z}=\mathrm{I}_{\alpha \beta} \mathrm{Z}^{\alpha} \mathrm{dZ} \mathrm{Z}^{\beta}=\epsilon^{\mathrm{A}^{\prime} \mathrm{B}^{\prime}} \pi_{\mathrm{A}^{\prime}} \pi_{\mathrm{B}^{\prime}}$ \\
\hline 2 & $\begin{array}{l}\text { 2-Dimensional Waving } \\
\text { Space } \\
\text { (for example Figure 4(b))) }\end{array}$ & $\begin{array}{l}2 \text {-form: } \\
\tau=\frac{1}{2} \mathrm{dL}\end{array}$ & $\tau=\frac{1}{2} \mathrm{I}_{\alpha \beta} \mathrm{d} \mathrm{Z}^{\alpha} \wedge \mathrm{dZ} Z^{\beta}=\in^{\mathrm{A}^{\prime} \mathrm{B}^{\prime}} \mathrm{d} \pi_{\mathrm{A}^{\prime}} \wedge \mathrm{d} \pi_{\mathrm{B}^{\prime}}$ \\
\hline & $\mathcal{H}$-Spaae & 3-form: $\theta$ & $\begin{aligned} \theta= & \frac{1}{6} \in_{\alpha \beta \gamma \delta} Z^{\alpha} d Z^{\beta} \wedge d Z^{\gamma} \wedge d Z^{\delta} \\
= & Z^{0} d Z^{1} \wedge d Z^{2} \wedge d Z^{3}-Z^{1} d Z^{0} \wedge d Z^{2} \wedge d Z^{3} \\
& +Z^{2} d Z^{0} \wedge d Z^{1} \wedge d Z^{3}-Z^{3} d Z^{0} \wedge d Z^{1} \wedge d Z^{2}\end{aligned}$ \\
\hline 4 & Spin Bundle & $\begin{array}{l}4 \text {-form: } \\
\phi=\frac{1}{4} \mathrm{~d} \theta\end{array}$ & $\begin{aligned} \phi & =\frac{1}{24} \epsilon_{\alpha \beta \gamma \delta} \mathrm{dZ}^{\alpha} \wedge \mathrm{dZ}^{\beta} \wedge \mathrm{dZ}^{\gamma} \wedge \mathrm{dZ}^{\delta} \\
& =\mathrm{dZ}^{0} \wedge \mathrm{dZ}^{1} \wedge \mathrm{dZ}^{2} \wedge \mathrm{dZ}^{3}\end{aligned}$ \\
\hline 5 & $\begin{array}{l}\text { Field Distorsion } \\
\text { Components }\end{array}$ & $\begin{array}{l}\text { Euler form: } \\
\begin{array}{l}\mathrm{Y}=\theta: \phi \\
\mathrm{da} \wedge \theta=\mathrm{Y}(\mathrm{a}) \phi\end{array}\end{array}$ & $\mathrm{Y}=\mathrm{Z}^{\alpha} \frac{\partial}{\partial \mathrm{Z}^{\alpha}}$ \\
\hline
\end{tabular}

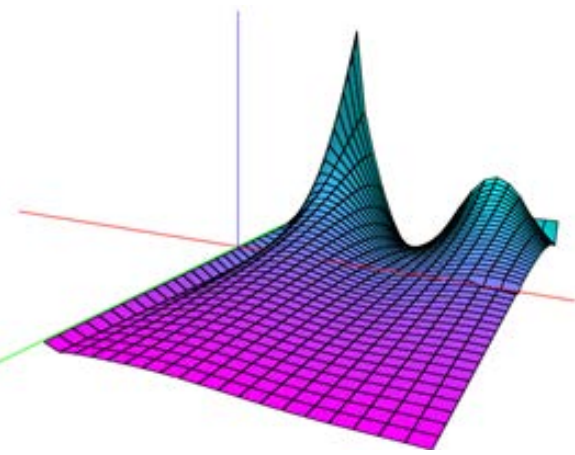

(a)

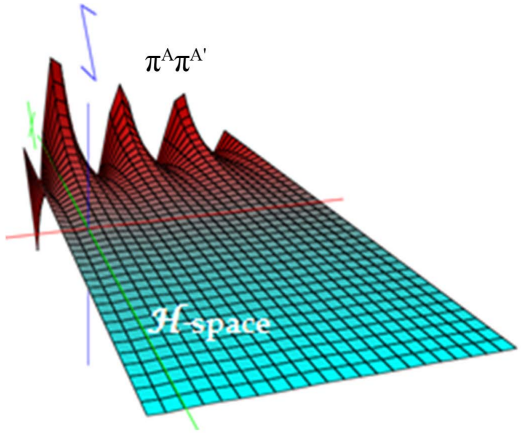

(b)

Figure 4. (a) Positive definite condition applied in the spinor $\omega_{\mathrm{A}} \omega_{\mathrm{B}}$, and $\pi^{\mathrm{A}} \pi^{\mathrm{A}^{\prime}}$, in the 2-dimensional model of the space-time kinematic twistor. In the model (b); is included the perturbations in the $\mathcal{H}$-space, that is to say, is the $\mathcal{H}$-space model of the twistor kinematic space.

$$
\mathrm{M}_{\mathrm{N}}^{2}=\frac{1}{2} \mathrm{~A}_{\alpha \beta} \overline{\mathrm{A}}^{\alpha \beta}
$$

which can be written on a 3-surface as:

$$
\mathrm{M}_{\mathrm{N}}^{2}=\frac{1}{2} \mathrm{~A}_{\alpha \beta} \overline{\mathrm{A}}^{\alpha \beta} \Sigma^{\alpha \alpha^{\prime}} \Sigma^{\beta \beta^{\prime}}
$$

Likewise, we find that the 3-surface twistor equation has a complex 4-dimensional family of solutions (a 3-surface twistor space $\pi(\Sigma)$ ) if and only if $\Sigma$, with their first and second fundamental forms are embedded in a conformallyflat space-time (see Figure 5).

The nature of the $\pi(\Sigma)$, from a point of view of QFT, are fermionic sources (currents) whose fermionic fields are Grassman numbers satisfying anti-commutation relations where bosonic fields and currents commute. 
We consider the following main result, which is the culmination of this research.

Theorem 3.1 (F. Bulnes, I. Verkelov, Y. Stropovsvky, I. Rabinovich). Spinor wave $\mathrm{S}^{\mathrm{AA}}$, in the non-commutative ring algebra (Clifford algebra type) has as Spec in the kinematic-twistor space-time as rotating embedding surfaces (waving) in the $\mathcal{H}$-space given by $\Pi_{\mathrm{AA}}$.

Proof. We consider the theorem 2.1, of the section 2, and considering the proposition 1. 2, to the contorted 2 -surfaces embedded with values in the $\mathcal{H}$ -space as $(\pi(S) \otimes \pi(S))^{*}$, we demonstrate that the deformed category of the moduli stack to the elements that acted in the space-time are the of non-commutative algebra whose spectrum ${ }^{6}$ (see the scheme of derived categories) is in the corresponding twistor kinematic space-time. This proves the asseveration of the theorem 3.1. The elements in the $\mathcal{H}$-space are bosonic fields commutating with currents. The waving is of type as Figure 6.

The moduli stack comes given by the gravitational waves given by the dualities between spinors and kinematic tensors.

These are gravitational waves in the space-time, since come of the torsion which is a second curvature, and by the arguments of fermion interactions and fermionic sources (and particle helicities), these produce torsion from microscopic level until the conforming of the macroscopic behaviour of the space-time near of massive source.

Then the evolution of the space from the Big Bang until the Universe that we know, have two periods of particles interacting, the first called leptogenesis where the Universe conforms the base of the fermions in different types of neutrinos. Then these new fermionic interacting and due to the particle helicities that go arising of the fermionic sources, generate the torsion modelled geomet-

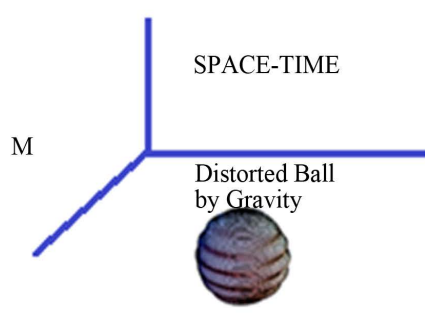

$\mathrm{T}(\mathrm{S})$

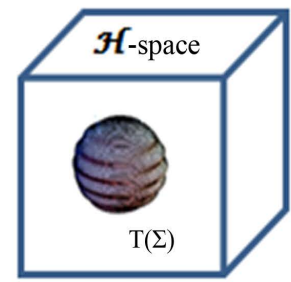
waves represented by spinors of energy

Figure 5. The appearing of the twistor image due to the twistor kinematic tensor acting on space-time from the twistor space $\pi(\Sigma)$.

${ }^{6}$ Theorem (I. Verkelov, F. Bulnes) [4] [5]. Considering the functors $\Phi, \Psi$, with the before properties Moduli $\underset{\mathrm{G \Psi}_{R}}{\stackrel{\Phi_{R} \mathrm{~F}}{\leftrightarrows}} \operatorname{Alg}_{\text {aug }}^{(n)}$, the corresponding homotopy equivalence, and their canonical homotopy, likewise, the relation $\operatorname{Alg}_{\text {aug }}^{(n)} \stackrel{\Phi^{-1}}{\cong} \operatorname{Moduli}_{n} \subseteq \operatorname{Fun}\left(\operatorname{Alg}_{\text {sm }}^{(n)}, \S\right)$, we have the following scheme

$$
\operatorname{Hom}_{\text {Moduli }}(X, \operatorname{Spec}(B)) \cong \operatorname{Hom}_{\operatorname{CAl}\left(\mathrm{Sp}_{\mathrm{p}}\right)}(B, \S) \text {. }
$$




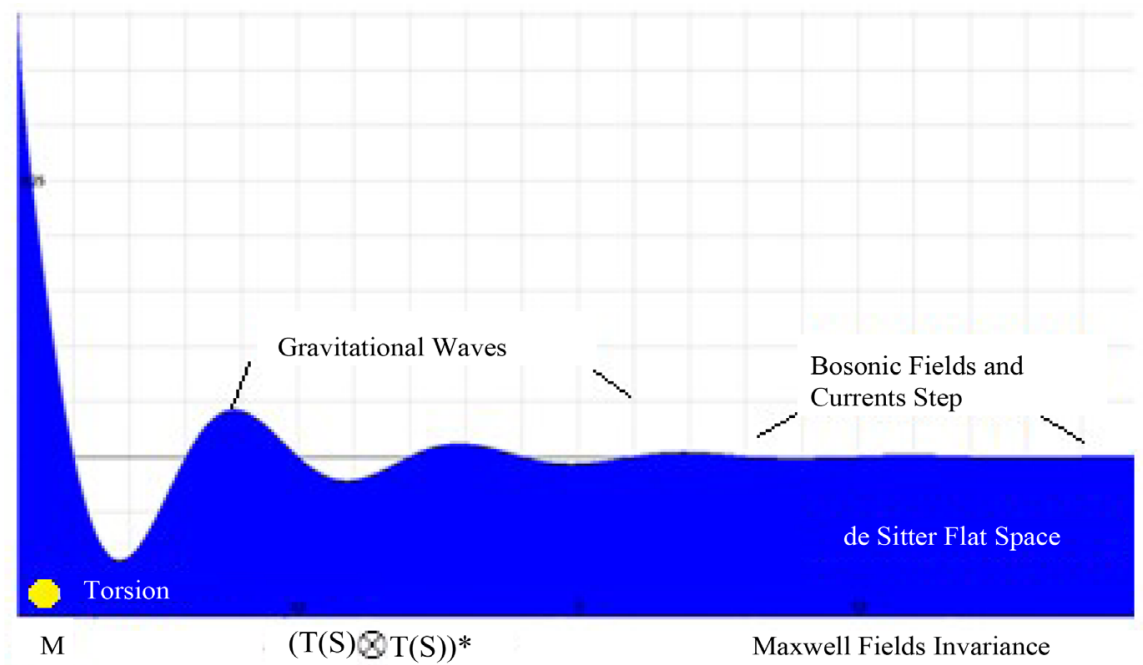

Figure 6. Bose-Einstein distribution to the gravitational waving evolution. The fermionic sources happened before of the flatness of the space-time far of the massive object $M$ (yellow source). The torsion component creates the waving on space-time.

rically by the space $\pi(\Sigma)$, then this produce a baryon-genesis whose action on the space-time produce the initial condiment of matter-energy, which finally gives the gravitational waves and after the bosonic fields and currents (see the Figure 7).

\section{Spectrum of Kinematic Tensor to Curvature and Design Curvature Censorships to Quantum Gravity Sensors}

Through consider the field study framework realized in this chapter we could determine and design a censorship condition with possibilities to their application in sensor technology [7].

In addition, we can consider the models of the space-time influenced for the fields on each particle of this, that is to say, consider the light cone of each particle intersects with the infinity nullity of the gravitational field that creates the deformation of the space-time [7].

In these intersections exist the detectable and measurable part that can be measured through microscopic electromagnetic fields and for the other side, that has the gravitational nature that provokes the curvature, generating enough energy to be bounded by the cosmic censorship of Penrose [8].

But the proper movements of the space-time from the 3-invariance in 4-dimensional complex space-time, and the expansion of the space-time studied in field theory frame considering gravitational fields, we can have the kinematic models given by the spaces that are asymptotically de Sitter and anti-de Sitter [9] [10]. These could give a fine censorship condition in the kinematic twistor models explained before.

Through a gauge field (electromagnetic type field as photons) acting on the background radiation of the Minkowski space MI, where the energy of the matter will be related with this gauge field through equation 


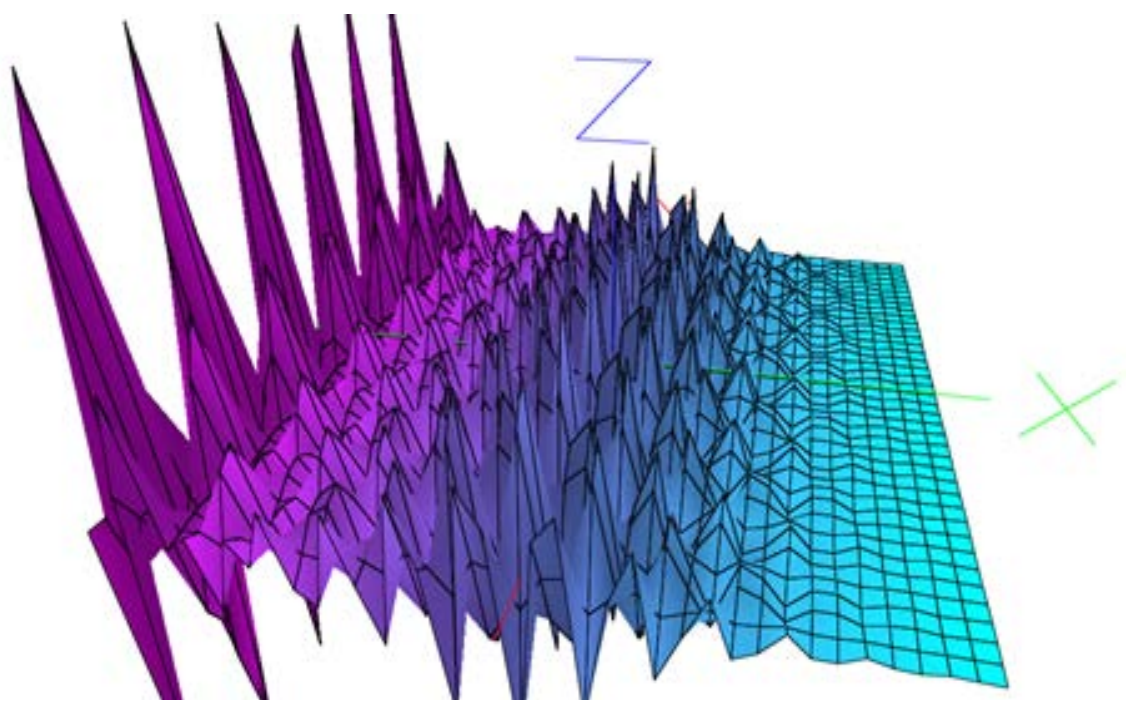

Figure 7. The torsion is represented in the clear purple waving [6] in the space-time.

$$
\mathrm{J}^{\alpha}=\mathrm{k}^{\alpha} \mathrm{T}^{\alpha \beta},
$$

(where $\mathrm{k}^{\alpha}$ ) can represent the density of background radiation which establishes for the curved part of the space (that in this case has spherical symmetry) together with the energy and matter tensor that (see the Figure 8)

$$
\frac{1}{4 \pi \mathrm{G}} \int_{\mathrm{S}^{2}} \mathrm{~T}_{\alpha \beta} \mathrm{k}^{\alpha} \mathrm{d} \sigma^{\beta} \geq \int_{\mathrm{S}^{2}} \mathrm{~J}^{\alpha} \mathrm{d} \sigma^{\beta} \geq 2 \pi \chi
$$

Then of the dominating energy condition normed by the twistor kinematic tensor given by the 3-dimensional ball affected (electromagnetic fields in $\mathrm{SU}(2)$, which is isomorphic to $\mathrm{S}^{3}$ ) by gravitation in the 4-dimensional space, we can to have the image of the twistor space of sphere in $(\pi(S) \otimes \pi(S))^{*}$, whose condition is had as:

$$
16 \pi \mathrm{M}^{2} \geq \mathrm{A},
$$

which is the Penrose censorship ${ }^{7}$ [11] for a singularity detected of spherical type [12] [13] [14] [15]. But from this idea can be designed and developed a sensor that use the torsion energy as second curvature energy. Because the fundamental conclusion of the end of the Section 3, is that the torsion energy obtained by movement of the 3 -dimensional ball inside the $\mathcal{H}$-space, is curvature energy and thus gravitational energy.

\section{Conclusion}

Curvature energy as image of the twistor kinematic-energy tensor applied to 3-dimensional sphere as surface to the cosmic censorship and the obtaining of curvature through gravitational waves can be very useful in quantum gravity theory to creation of advanced sensor devices that can measure the deformation

$$
7\left(\int_{S^{2}} \Omega\left(1-\nabla^{2} \log \Omega\right)\right)^{2} \geq 4 \pi \int \Omega^{2} \text {. }
$$




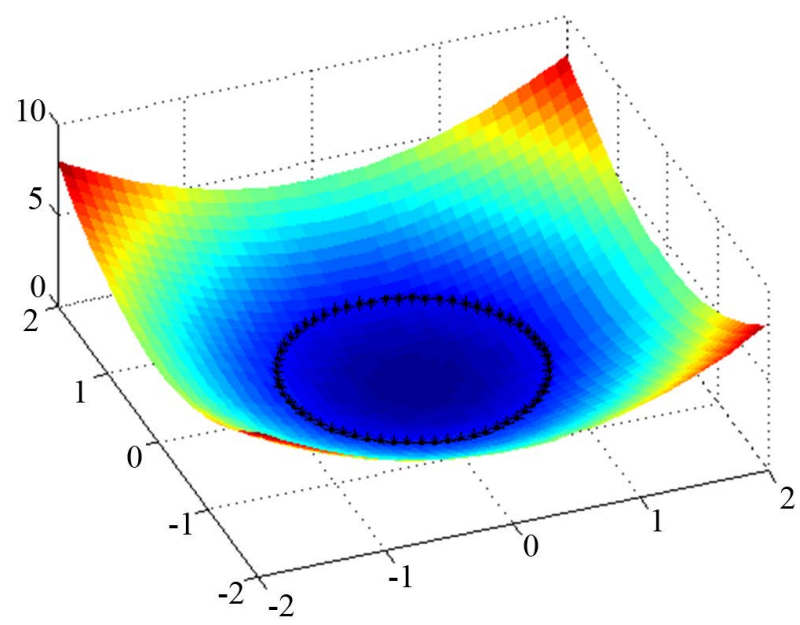

Figure 8. Degenerated neutrinos energy, which will be persistent to space-time background, being a strong indicium of the torsion (rest of the leptogenesis/baryongenesis process. This low energy signed in the circle is the represented in the right extreme of the inequality (27). Then gravitational waves appear.

on surfaces affected to micro-local level by the energy-matter-momentum tensor variations. One of these variations is the torsion energy, which is the curvature energy. Likewise, theoretically the integral representation given to the electrical charge depending of the momenta, establishes through analysis realized in duality that the gravitational energy condition required is to detect curvature in terms of energy. This is obtained with a censorship condition on cylindrical gravitational waves. These gravitational waves are produced from a 3-dimensional sphere located inside the background model of the space-time, whose values are in the space $(\pi(S) \otimes \pi(S))^{*}$. This obeys to a topological space as complex Riemannian manifold with local structure, which is isomorphic to a Hilbert space to this dominated energy in the space. Then the energy condition in this case is established for the existence of the sources, which is given by gravitational waves (source detection). These gravitational waves are solutions of a twistor equation whose spinor equivalent is the solution to the dominated energy by the presence of matter of a massive object whose existence in the space-time is given by this energy condition [13] [14]. This study is bounded to the curvature energy extended to the field torsion, using the spinor technology to create waves from field interactions. Studies realized on dilatons used as gauge particles to measure gravitational distortions has been proposed in several works [6] [8]. The idea of use fields to measure other fields is extended to other field formalisms considering tropical geometries in a complex Riemannian model of the space-time, that they can be carried to the technological design of sensor devices to detect quantum gravity [16]. The following step will be the application of the $\mathcal{H}$-states [7] [16] to produce that technology.

\section{Acknowledgements}

I am very grateful with Rene Rivera Roldan, Eng, Head of Electronic Engineer- 
ing Division for your support to give facilities in time and electronic laboratory devices to the research. Also, I am very grateful with Evaristo Vázquez, C. B, Finance Department, TESCHA, and Edgar Daniel Sánchez Balderas, L. B, General Director of TESCHA, for the financing support.

\section{References}

[1] Penrose, R. and Rindler, W. (1986) Spinors and Space-Time, Vol. 2. Cambridge University Press. https://doi.org/10.1017/CBO9780511524486

[2] Esposito, G. (1992) Fortschritte der Physik/ Progress of Physics, 40, 1-30. https://doi.org/10.1002/prop.2190400102

[3] Kobayashi, K. and Nomizu, K. (1969) Foundations of Differential Geometry. Vol. 2, Wiley and Sons, New York.

[4] Verkelov, I. (2014) Pure and Applied Mathematics Journal. Special Issue: Integral Geometry Methods on Derived Categories in the Geometrical Langlands Program, 3, 12-19. https://doi.org/10.11648/j.pamj.s.2014030602.13

[5] Bulnes, F. (2017) Integral Geometry Methods in the Geometrical Langlands Program, SCIRP, USA.

[6] Guivenchy, E. (2015) Journal on Photonics and Spintronics, 4, 14-22.

[7] Bulnes, F. (2017) Detection and Measurement of Quantum Gravity by a Curvature Energy Sensor: H-States of Curvature Energy, Recent Studies in Perturbation Theory. In: Uzunov, D., Ed., InTech,

https://www.intechopen.com/books/recent-studies-in-perturbation-theory/detectio n-and-measurement-of-quantum-gravity-by-a-curvature-energy-sensor-h-states-of -curvature-ener https://doi.org/10.5772/68026

[8] Bulnes, F. (2012) Journal of Electromagnetic Analysis and Applications, 4, 252-266. https://doi.org/10.4236/jemaa.2012.46035

[9] Gibbons, G.W. (1984) The Isoperimetric and Bogomolny Inequalities for Black Holes. In: Willmore, T.J. and Hitchin, N.J., Eds., Global Riemannian Geometry, Ellis Horwood, Halsted Press, Chichester, New York, 194-202.

[10] Geroch, R. (1977) Asymptotic Structure of Space-Time. In: Esposito, F.P. and Witten, L., Eds., Asymptotic Structure of Spacetime, Proceedings of a Symposium on Asymptotic Structure of Space-Time (SOASST), University of Cincinnati, Ohio, 14-18 June 1976, 1-105. https://doi.org/10.1007/978-1-4684-2343-3_1

[11] Kelly, R.M. (1985) Asymptotically Anti-de Sitter Space-Time. Twistor Newsletter, No. 20, 11-23.

[12] Frauendiener, J. (2001) Physical Review Letters, 87, Article ID: 101101. https://doi.org/10.1103/PhysRevLett.87.101101

[13] Penrose, R. (1982) Proceedings of the Royal Society of London A, 381, 53-63.

[14] Tod, K.P. (1985) Classical and Quantum Gravity, 2, L65-L68.

[15] Dougan, A.J. (1992) Classical and Quantum Gravity, 9, 2461-2475. https://doi.org/10.1088/0264-9381/9/11/012

[16] Bulnes, F. (2014) Design of Quantum Gravity Sensor by Curvature Energy and Their Encoding. Science and Information Conference, London, 27-29 August 2014, 855-861. 
Submit or recommend next manuscript to SCIRP and we will provide best service for you:

Accepting pre-submission inquiries through Email, Facebook, LinkedIn, Twitter, etc. A wide selection of journals (inclusive of 9 subjects, more than 200 journals)

Providing 24-hour high-quality service

User-friendly online submission system

Fair and swift peer-review system

Efficient typesetting and proofreading procedure

Display of the result of downloads and visits, as well as the number of cited articles Maximum dissemination of your research work

Submit your manuscript at: http://papersubmission.scirp.org/

Or contact jmp@scirp.org 